\title{
Bismuth quantum-wires arrays fabricated by electrodeposition in nanoporous anodic aluminum oxide and its structural properties
}

\author{
Yong Peng ${ }^{\mathrm{b}}$, Dong-Huan Qin ${ }^{\mathrm{a}}$, Rong-Jie Zhou ${ }^{\mathrm{a}, \mathrm{b}}$, Hu-Lin Li ${ }^{\mathrm{a}, \mathrm{b}, *}$ \\ a Department of Chemistry, Lanzhou University, Lanzhou 730000, Peoples' Republic of China \\ b Applied Magnetics Key Laboratory of the Ministry Education, Lanzhou University, Lanzhou 730000, Peoples' Republic of China
}

Received 21 April 2000; accepted 12 June 2000

\begin{abstract}
Ultrafine bismuth nanowire arrays have been fabricated by electrodeposition into nanoporous anodic aluminum templates in a composite electrolyte solution, in which the diameter of nanowire as low as $3 \mathrm{~nm}$ can be obtained. The micrographs and crystal structures of Bi nanowires are studied by transmission electron microscopy (TEM), selected-area electron diffraction (SAED), and $\mathrm{X}$-ray diffraction (XRD). It is found that each nanowire is essentially a single crystal and has a different orientation in an array. The optical properties of the Bi nanowire arrays are studied by UV-VIS spectra. The experimental results exhibit blue-shifted phenomena with decreasing wire diameter, which is qualitatively consistent with the effective medium theory. (C) 2000 Elsevier Science S.A. All rights reserved.
\end{abstract}

Keywords: Quantum-wires arrays; Aluminum oxide; Bismuth nanowire

Semimetallic bismuth is a material exhibiting interesting magnetoresistance (MR) characteristics and finitesize effects, whose electronic properties are fundamentally different from those of common metals due to the complex and highly anisotropic Fermi surface [1-7]. Especially, ultrafine bismuth nanowires arrays are considered a good candidate to study quantum confinement effect, which is desirable for wide-field sensing [8] and thermoelectric materials application [9]. Therefore, recently considerable interest in fabricating and studying properties of $\mathrm{Bi}$ nanowires or arrays of nanowire [1] [8-14]. The fabrications of arrays of $\mathrm{Bi}$ nanowires are synthesized by electrodeposition in polycarbonate membranes [1,8] or particle track-etched membranes (PTMs) [11], and injecting its liquid melt or a vapor-phrase technique into a porous $\mathrm{Al}_{2} \mathrm{O}_{3}$ template $[9,10,12,13]$. However, the attribution of pores of polycarbonate membrane is not uniform, which can not be used to fabricate the high quality arrays of $\mathrm{Bi}$ nanowires. The pressure injection method or a vaporphrase technique is difficult to fabricate the pore diameter of bismuth nanowires below $10 \mathrm{~nm}$. And this

\footnotetext{
* Corresponding author.
}

method is highly costly, and complex in operation. If the fabrication by electrodeposition into nanoporous anodic aluminum oxide (AAO) can be carried out, the shorts can been avoided. To the best of the authors' knowledge, the arrays of $\mathrm{Bi}$ nanowires were not prepared by electrodeposition into nanoporous AAO films. The possible reason is that the bismuthal salt is very easy to hydrolyze. When the solution $\mathrm{pH}$ is controlled under 0.9 or lower, the nanoporous AAO templates are dissolved in solution.

When anodized in an acidic electrolyte, aluminum forms a porous oxide with very uniform and parallel pores open at one end and sealed at the other [15-18]. Its structure is described as a closed-packed array of columnar cells, each containing a central pore of which the side and interval can be controlled by changing the forming conditions. In recent years, there has been interest in this material as a medium for creating uniform nanostructures since its pores can function as 'nanotemplates' in which small metal and semiconductor particles can be electrochemically deposited [15-22].

Here, the Bi quantum-wire arrays were electrodeposited in nanoporous AAO membranes by a novel deposition electrolyte solution which was prepared. The technique successfully carried out the fabrication of $\mathrm{Bi}$ 

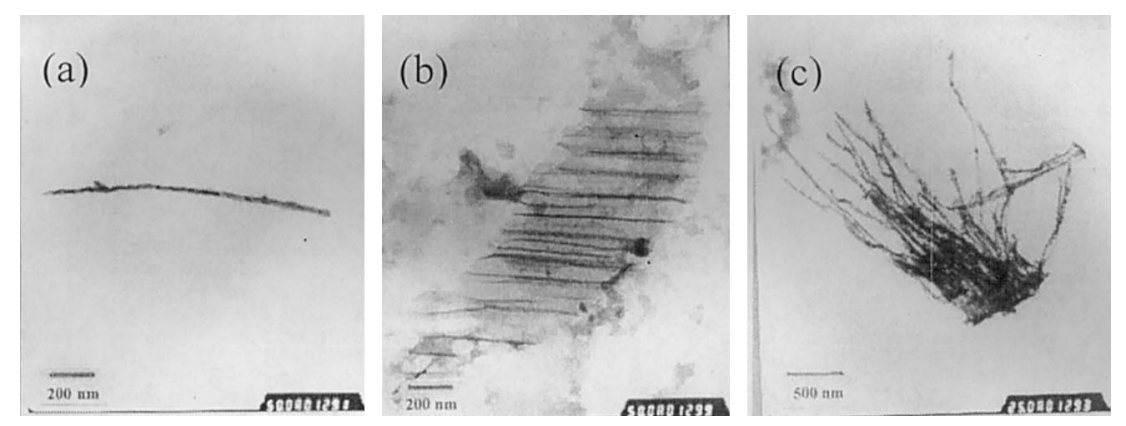

Fig. 1. Transmission electron microscopy (TEM) micrographs of Bi nanowires, (a) a single of Bi nanowire; (b) Bi nanowires paralleled in banks; (c) a bundle of Bi nanowires.

nanowires arrays with cost-effectiveness, simplicity in operation, and the ability of deposition into templates, whose diameter was in the range of $3-1000 \mathrm{~nm}$. Meanwhile the results obtained by transmission electron microscopy (TEM), selected-area electron diffraction (SAED), X-ray diffraction (XRD), and UV-VIS-NIR absorption spectra of $\mathrm{Bi}$ quantum-wires in nanoporous AAO films (Bi/AAO) were presented. Their magnetoresistance properties will be reported in a subsequent research paper.

The nanoporous template was fabricated by anodizing an electrochemically polished high purity aluminum substrate $(99.999 \%)$ in different concentration oxalic acid solution or sulfuric acid solution as the electrolyte. In the work, films were prepared with pore diameters of ca. $60,40,25,15 \mathrm{~nm}$ (anodized in oxalic acid); ca. $5 \mathrm{~nm}$ (anodized in sulfuric acid). When the voltage of anodic oxidation was relatively high, the technique of decreasing voltage step by step was adopted, which made the electrodeposition easier. The micrographs and structures of nanoporous AAO templates were reported by the authors previously $[16,17,22]$.

The resulting AAO membranes were used as templates for the Bi nanowires. Following anodization $\mathrm{Bi}$ deposition was carried out by using alter current (AC) electrodeposition in an aqueous solution of bismuth chloride. The electrolyte contained $0.15 \mathrm{~mol} \mathrm{l}^{-1}$ bismuth chloride, $0.3 \mathrm{~mol} \mathrm{1}^{-1}$ tartaric acid, and $100 \mathrm{~g}^{-1}$ glycerol. The resulting cloudy solution was buffered to pellucid with $37 \mathrm{~mol}^{-1}$ hydrochloric acid. In order to keep the solution adjusted, $13 \mathrm{~mol} 1^{-1}$ aqueous ammonia was used to control the $\mathrm{pH}$ 3.0. The electrolysis was conducted at $15^{\circ} \mathrm{C}, 200 \mathrm{~Hz}$, different deposition time and different $\mathrm{AC}$ voltage. One pledged that the nanowire lengths of each sample were longer than 1.5 $\mu \mathrm{m}$ in this work. Next, the Al substrates were etched away by an amalgamation process.

To confirm that the nanochannel arrays have been fabricated by continuous dense Bi nanowires, the aluminum matrix was dissolved in $0.1 \mathrm{M} \mathrm{NaOH}$ aqueous solution, which did not attack the Bi nanowires. Fig. 1 shows three representative TEM images of $\mathrm{Bi}$
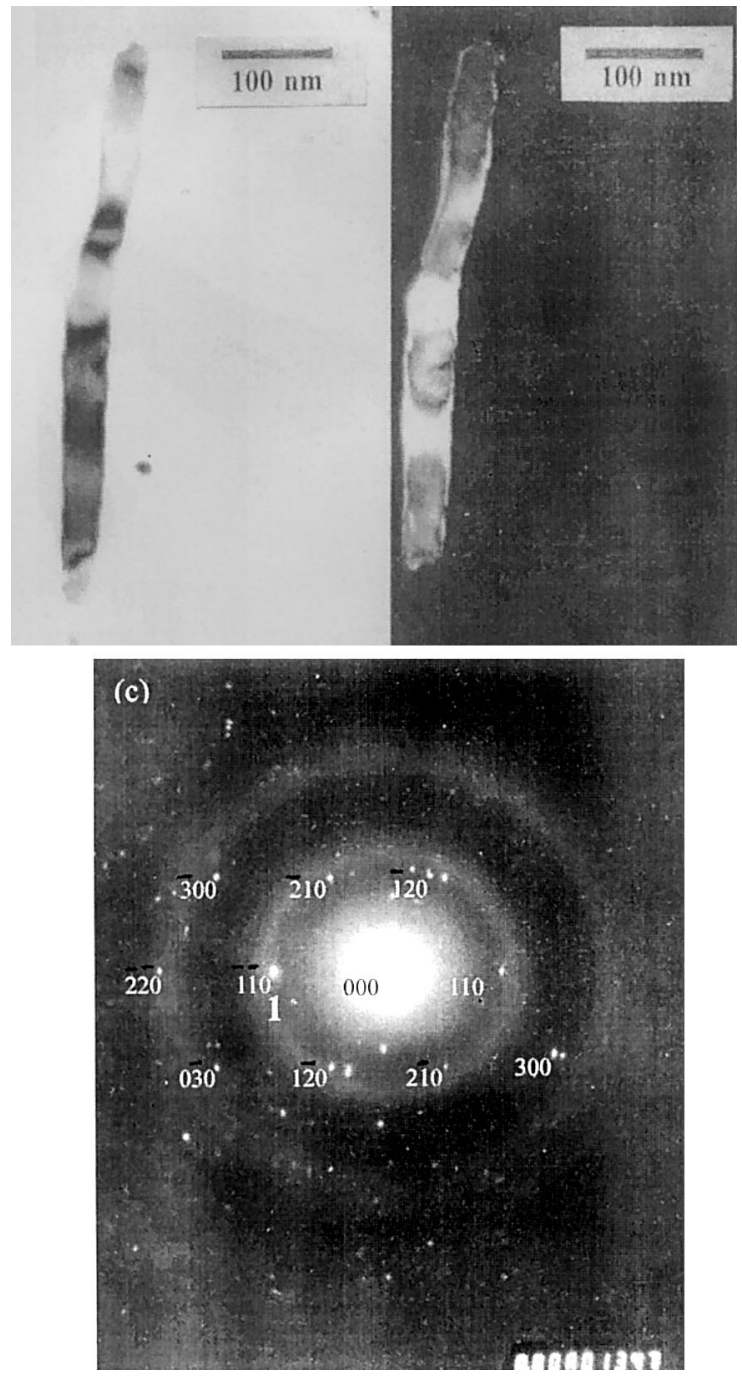

Fig. 2. Transmission electron microscopy (TEM) micrographs of a single Bi nanowire with average diameter of $40 \mathrm{~nm}$. (a) Bright-field; (b) dark-field of the same Bi nanowire from the spot 1 or (11) from (c). (c) Electron diffraction patterns of the same Bi nanowire from the [001] orientation. The inset data is the cubic indices of the diffractive spots. 


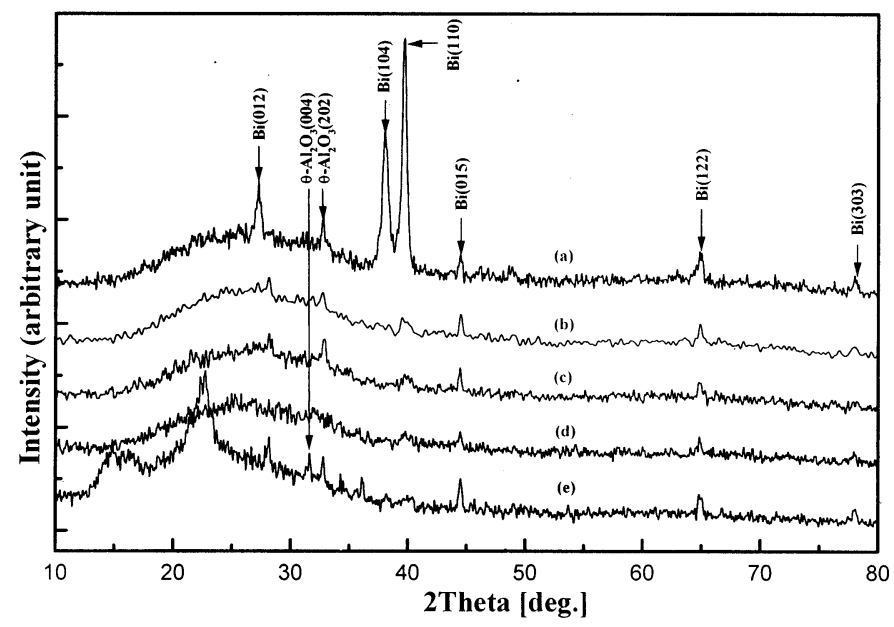

Fig. 3. X-ray diffraction (XRD) patterns of Bi nanowire arrays with average wire diameter of (a) $60 \mathrm{~nm}$, (b) $40 \mathrm{~nm}$, (c) $25 \mathrm{~nm}$, (d) $15 \mathrm{~nm}$, (e) $5 \mathrm{~nm}$.

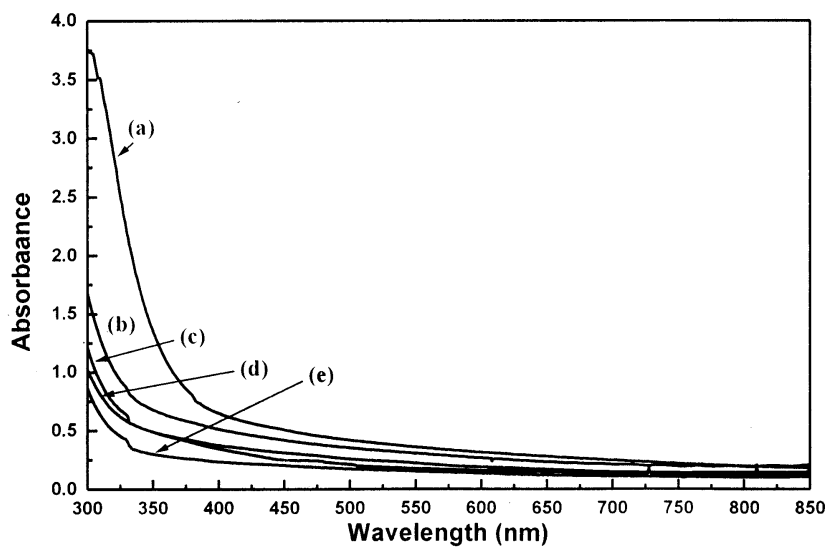

Fig. 4. Optical absorption spectra of Bi nanowire arrays with average wire diameter of (a) $60 \mathrm{~nm}$, (b) $40 \mathrm{~nm}$, (c) $25 \mathrm{~nm}$, (d) $15 \mathrm{~nm}$, (e) $5 \mathrm{~nm}$.

nanowires. A single Bi nanowire is shown in Fig. 1(a), in which the alumina matrix is dissolved completely. Fig. 2(b) shows that the Bi nanowires are parallel in banks when the alumina matrix is greatly dissolved, whose situations within the pores of the nanoporous alumina matrix are kept. Fig. 1(c) shows a bundle of Bi nanowires, whose bottoms adhere to each other, making it difficult to resolve the individual wires. The reason is that the aluminum matrix is partially dissolved from the bottom of the substrate. And one can see that the Bi nanowires are ductile, which indicated their excellent crystallinity.

As is known, the electron diffraction experiments of single crystal must involve to the bright, dark field and electron diffraction patterns. Shown in Fig. 2 are the results of the SAED pattern, which reveals that the single $\mathrm{Bi}$ nanowire is a single crystal (the accelerated voltage of electron is $100 \mathrm{kV}$ in this experiment). Fig. 2(a) is the bright field image of the single Bi nanowire, which is selected in the SAED experiments. And Fig. 2 (b) is the dark field image of the same single $\mathrm{Bi}$ nanowire from the diffractive spot 1 or (110) (Fig. 2(c)). One can see that the two photos show the same nanowire. At the same time the result of the electron diffraction pattern of this single nanowire is obtained in Fig. 2(c). From this photo, one can see that the diffractive spots can be organized in an almost precise hexagon or parallelogram, which reveals that the rhombohedral lattice structure of bulk $\mathrm{Bi}$ is also preserved in the nanowires. Fig. 2(c) shows a [001] diffraction pattern of Bi nanowire. According to the geometry analyses of electron diffractogram, the cubic indices of the diffractive spots in the electron diffraction pattern are demarcated (Fig. 2(c)). From Fig. 3(c), one can also see three bright rings which result from carbon film used to support a sample in TEM experiments. To confirm the experiment results, other $\mathrm{Bi}$ nanowires are selected to perform the SAED experiments and obtain the same results. Therefore, it can be proposed that each single Bi nanowire is a single crystal.

Fig. 3 shows the XRD patterns of Bi/AAO films removed from the Al substrate. It can be seen that all the XRD peaks are located very close to the peak positions of bulk $\mathrm{Bi}$, revealing that the rhombohedral lattice structure of bulk $\mathrm{Bi}$ is also preserved in the nanowires. At the same time it can be seen that the dominating peak of $\mathrm{Bi}$ nanowires is not the same that of bulk $\mathrm{Bi}(012)$, and the relative intensities of peaks of $\mathrm{Bi}$ nanowires have departures from those of bulk Bi when the diameter of $\mathrm{Bi}$ nanowires decreases from (a) to (d). For the sample with an average diameter of ca. $60 \mathrm{~nm}$ only six peaks were observed (012), (104), (110), (015), (122), (303), which keep a similar relative intensity of the peaks like bulk Bi but the dominating peak. Fig. 3(b)-(d) show that the Bi (104) peak has almost disappeared and the peaks of (012), (110), (015), (122), (303) remain. The relative intensities of the left peaks are completely different from those of the peaks of bulk $\mathrm{Bi}$. The experiment results are different from those of Zhang [10], where the arrays of Bi nanowires are fabricated by pressure injection process. It can be deduced that the reason maybe is that the crystallization between the process of electrodeposition and that of pressure injection is different. From the XRD results, the arrays of $\mathrm{Bi}$ nanowires show polycrystalline structure as if the results conflict with the results of SAED above. Considering it is the statistical results obtained by the XRD pattern and the diffraction pattern of different grains indicate different orientations, it can be proposed that these individual $\mathrm{Bi}$ nanowires are essentially single crystal and each $\mathrm{Bi}$ nanowire in an array has a different crystal orientation.

Fig. 4 shows the optical absorption spectra of these Bi nanowires composite membrane. As reported above, it is pledged that the length of each sample is longer 
than $1.5 \mu \mathrm{m}$. So one can ignore the optical influence that the length induces. One can see all the samples of composite membrane have an absorption edge at the short wavelength and the absorption edge is blueshifted from (a) to (e) in Fig. 4 with decreasing wire diameter, which is qualitatively consistent with the effective medium theory. This result is different from that obtained by Zhang et al. [10]. To confirm the result, another similar series of samples are performed and the same results are obtained, which shows there is not a dramatic change in the band gap energy of the $\mathrm{Bi}$ nanowires as a function of wires diameter.

In summary, we have successfully fabricated the arrays of $\mathrm{Bi}$ nanowires by electrodeposition into nanoporous AAO templates, which accomplishes the fabrication of $\mathrm{Bi}$ nanowires arrays with cost-effectiveness, simplicity in operation, and the ability of deposition into templates, diameter in the range of 3-1000 $\mathrm{nm}$. The studies of the micrographs and crystalloid structure propose that each nanowire is a single crystal and has different orientation in an array. And optical properties of $\mathrm{Bi}$ nanowire arrays are blue-shifted with decreasing wire diameter, which is qualitatively consistent with the effective medium theory.

\section{Acknowledgements}

This work is supported by Nation Natural Science Foundation of China. We would like to express our sincere thanks to Chief Engineer Gui-Xun Cao of Gansu Instrumental Analysis \& Research Center for TEM measurement and analysis of Electron Diffraction result, to Chief Engineer Da-Kang Song of Material Department of Lanzhou University for measurement and analysis of XRD data. We also appreciate Dr Xiong Chen, who is from State Key Laboratory of Arid Agroecology, for UV-VIS measure.

\section{References}

[1] K. Liu, C.L. Chien, P.C. Searson, Phys. Rev. B 58 (1998) R14681.

[2] M. Lu, R.J. Zieve, A. Van Hulst, H.M. Jaeger, T.F. Rosenbaum, S. Radelaar, Phys. Rev. B 53 (1996) 1609.

[3] J.H. Mangez, J.P. Issi, J. Heremans, Phys. Rev. B 14 (1976) 4381.

[4] S. Golin, Phys. Rev. 166 (1968) 643.

[5] N. Garcia, Y.H. Kao, M. Strongin, Phys. Rev. B 5 (1972) 2029.

[6] R.D. Brown, R.L. Hartman, S.H. Koenig, Phys. Rev. 172 (1968) 598.

[7] F.Y. Yang, K. Liu, K.M. Hong, D.H. Reich, P.C. Searson, C.L. Chien, Science 284 (1999) 5418.

[8] K. Liu, C.L. Chien, P.C. Searson, K.Y. Zhang, Appl. Phys. Lett. 73 (1998) 1463.

[9] J. Heremans, C.M. Thrush, Phys. Rev. B 59 (1999) 12579.

[10] Z.B. Zhang, D. Gekhtman, M.S. Dresslhaus, J.Y. Ying, Chem. Mater. 11 (1999) 1659.

[11] L. Piraux, S. Dubois, J.L. Duvail, A. Radulescu, S. DemoustierChampagne, E. Ferain, R. Legras, J. Mater. Res. 14 (1999) 3042.

[12] Z.B. Zhang, X.Z. Sun, M.S. Dresselhaus, J.Y. Ying, J.P. Heremans, Appl. Phys. Lett. 73 (1998) 1589.

[13] J. Heremans, C.M. Thrush, Z.B. Zhang, X. Sun, M.S. Dresselhaus, J.Y. Ying, D.T. Morelli, Phys. Rev. B 58 (1998) R10091.

[14] J.L. CostaKramer, N. Garcia, H. Olin, Phys. Rev. Lett 78 (1997) 4990.

[15] C.A. Huber, T.E. Huber, M. Sadoqi, J.A. Lubin, S. Manalis, C.B. Prater, Science 263 (1994) 800.

[16] Y. Peng, Hao-Li Zhang, Shan-Lin Pan, H.L. Li, J. Appl. Phys (published).

[17] S.L. Pan, H.L. Zhang, Yong Peng, Zhen Wang, H.L. Li, Chem. J. Chin. Univ. 20 (1999) 1622.

[18] D. Routkevitch, A.A. Tager, J. Haruyama, D. Almawlawi, M. Moskovits, J.M. Xu, IEEE. Trans. Electron. Dev. 43 (1996) 1646.

[19] C.W. Wang, Y. Peng, H. Li. Zhang, H.L. Lin, Acta Phys. Sinica (in China), 11 (1999) 2146.

[20] G.H. Pontifex, P. Zhang, Z. Wang, T.L. Haslett, D. Almawlawi, M. Moskovits, J. Phys. Chem. 95 (1991) 9989.

[21] Yong Peng, Rong-Jie Zhou, Hulin Li, Zhao-Jie Chen, Micrographies and Crystalloid Structures and Magnetic Properties of Co Nanowires Arrays Deposited in Alumina Template, Chinese Science Bulletin. J. Appl. Phys. 87 (2000) 7405.

[22] Yong Peng, Rong-Jie Zhou, Hu-Lin Li, 6th China-Janpan Bilateral Symposium on Interlligent Electrophotonic Materials and Molcular Electronic, Beijing, China, Dec 3-4, 1999, Micrographies and Crystalloid Structures of Co Nanowires Deposited in Alumina Film. 